\title{
Hepatic manifestations of familial patent ductus venosus in adults
}

\author{
S Jacob, G Farr, D De Vun, H Takiff, A Mason
}

\begin{abstract}
Background-The ductus venosus connects the umbilical vein to the inferior vena cava during fetal life and subsequently closes rapidly after birth. It is known as patent ductus venosus when it remains patent in adulthood.

Patients-A 43 year old man with a history of panhypopituitarism presented with recurrent bouts of pedal oedema associated with fatigue, hypoalbuminaemia, and elevated prothrombin time. An ultrasound examination of his abdomen with Doppler revealed notable attenuation of the main portal vein with diminished intrahepatic branches; a computed tomography scan with angiography revealed a large collateral vein within the liver consistent with a patent ductus venosus. Sequential liver biopsies showed a considerable reduction in the calibre and number of the portal veins. His younger brother, who was diagnosed with alcohol related cirrhosis, suffered from intermittent bouts of encephalopathy and was found to have the same vascular lesion. A third brother was found to have a patent ductus venosus as well as two large hepatic masses consistent with focal nodular hyperplasia.

Conclusion-The syndrome of familial patent ductus venosus has only previously been described in three infant brothers who presented with hepatic encephalopathy and fatty degeneration of the liver. This report documents three brothers with a patent ductus venosus presenting in adulthood with different manifestations of liver disease. The presence of the same vascular anomaly in three brothers is highly suggestive of a recessive genetic trait with an anatomical manifestation of patent ductus venosus.

(Gut 1999;45:442-445)
\end{abstract}

Keywords: patent ductus venosus; portal vein sclerosis; familial; hepatic synthetic dysfunction; hepatic encephalopathy

The ductus venosus connects the umbilical vein to the inferior vena cava during fetal life and subsequently closes rapidly after birth. Reports have documented intrahepatic portosystemic venous communications from the portal venous system to the inferior vena cava which have been attributed to a patent ductus venosus, the sequelae of liver biopsy, or even the pathological effects of cirrhosis. ${ }^{1-12}$ Patent ductus venosus has been detected in infants with and without cardiac defects. ${ }^{1-4}$ In adults, the diagnosis is made as an incidental finding or in subjects presenting with hypoglycaemia or portosystemic encephalopathy. ${ }^{5-12}$ Recently, a report of three brothers presenting in childhood with encephalopathy, fatty degeneration of the liver, and abnormal hepatic synthetic function suggested that development of patent ductus venosus may have a genetic component. ${ }^{3}$ In agreement, we describe here familial patent ductus venosus in three brothers presenting in adulthood with differing manifestations of chronic liver disease.

\section{Case reports}

PATIENT 1

A 43 year old man was referred to Ochsner clinic in 1995 with a diagnosis of idiopathic oedema, pretibial cellulitis, and fatigue. On examination, he had palmar erythema, but no other signs of chronic liver disease. His liver was palpated one finger breadth below the right costal margin and there was no evidence of ascites or splenomegaly. Bilateral pitting oedema was observed and he had evidence of a well circumscribed pretibial cellulitis on his right leg. Results of laboratory studies were as follows: white blood cell count, $12.3 \times 10^{9} / 1$; haemoglobin, $150 \mathrm{~g} / 1$; platelet count, $163 \times$ $10^{9} / 1$; serum aspartate aminotransferase, 22 $\mathrm{U} / 1$; alanine aminotransferase, $29 \mathrm{U} / 1$; albumin, $27 \mathrm{~g} / 1$; total bilirubin, $6.8 \mu \mathrm{mol} / 1$; alkaline phosphatase, $110 \mathrm{U} / 1$; and a prolonged prothrombin time of 14 seconds. His ammonia concentration was normal at $26 \mu \mathrm{mol} / 1$.

In 1980, a diagnosis of panhypopituitarism was made when he presented with manifestations of hypothyroidism, hypotestosteronism, and adrenal insufficiency. These symptoms were adequately managed with hormone replacement therapy and although no cause for this problem was detected, a recent magnetic resonance imaging (MRI) scan revealed calcification in the hypothalamus. In 1990 he presented with renal colic and was diagnosed with bilateral nephrolithiasis with hypercalciuria. Since then he has suffered from recurrent episodes of passing renal stones. His parathormone concentration was $15 \mathrm{pg} / \mathrm{ml}$ which was within normal limits. Subsequently in 1992, he developed hypoalbuminaemia and pedal oedema without evidence of proteinuria. An abdominal ultrasound at that time showed diminished right and left portal veins with hepatofugal flow in the main portal vein. His liver biopsy was reported as normal with disturbed hepatic architecture.

Abbreviations used in this paper: CT, computed tomography; HFE, haemochromatosis gene; MRI, magnetic resonance imaging. 


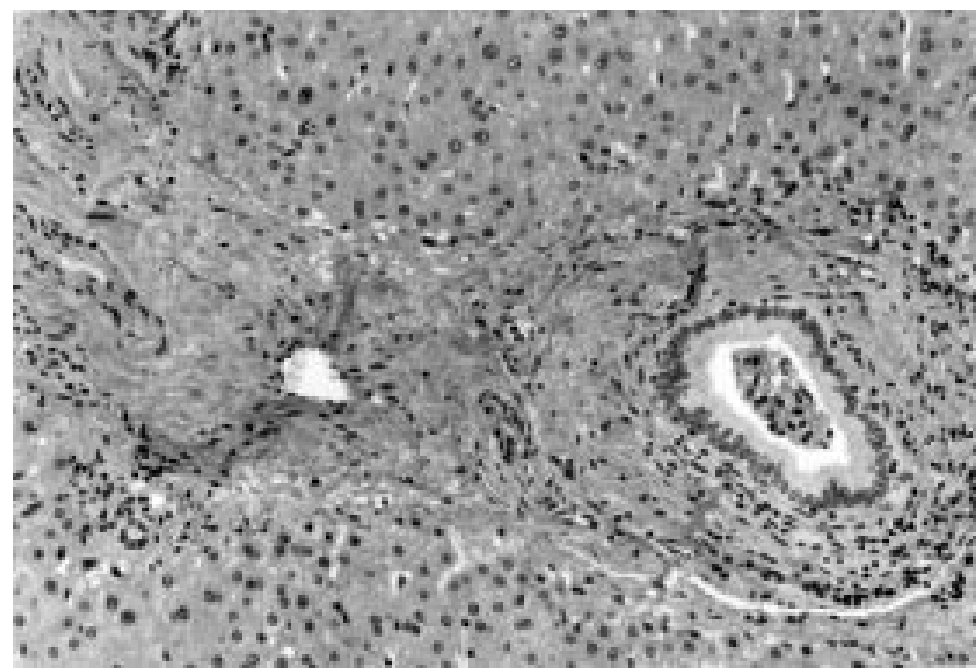

Figure 1 Microscopic section of the liver in patient 1, showing a sclerosed portal vein on the left of the portal triad (haematoxylin and eosin; original magnification $\times 100$ ).

On this admission, a follow up liver biopsy revealed normal hepatic parenchyma without evidence of steatosis, inflammation, or fibrosis. However, there was a notable reduction in the calibre of the portal veins compared with his liver biopsy performed three years previously.
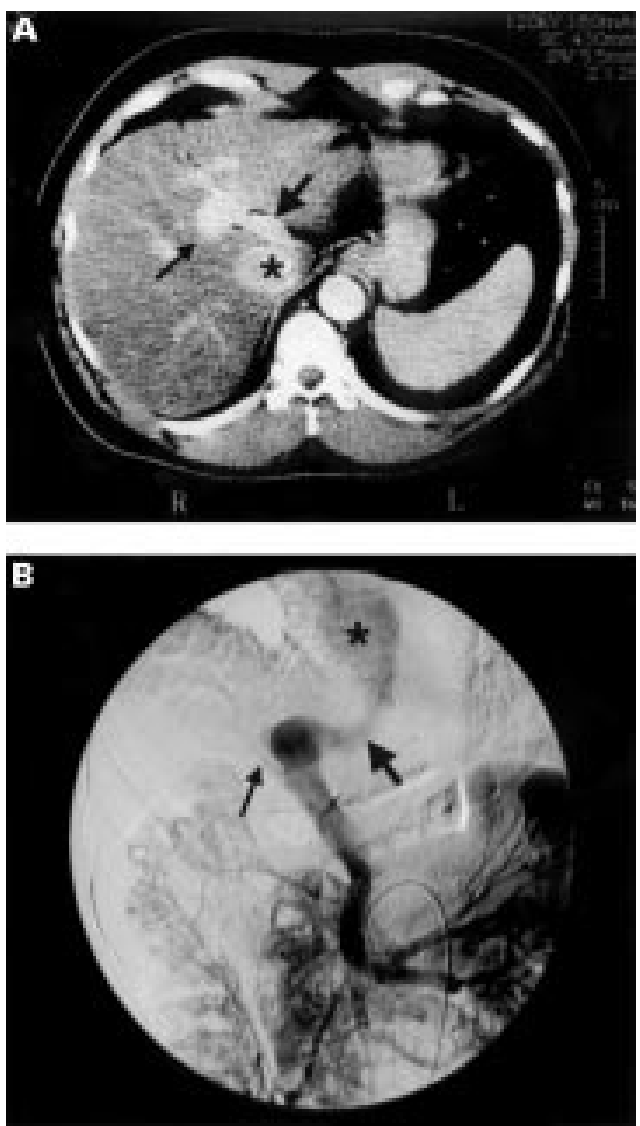

Figure 2 (A) Computed tomography of the abdomen of patient 1 during infusion of contrast into the superior mesenteric artery, showing the patent ductus venosus. The thin arrow points to the portal vein, the thick arrow to the patent ductus venosus, and the asterisk marks the inferior. vena cava. (B) Selective angiogram of superior mesenteric artery of patient 1 , showing the patent ductus venosus in the venous phase. The thin arrow points to the small residual right portal vein, the thick arrow to the patent ductus venosus, and the asterisk marks the right atrium.
The portal vein was sclerosed and half the diameter of the bile duct (fig 1). The ultrasound examination of the liver showed notable attenuation of the main portal vein at the level of the porta hepatis with extremely small intrahepatic branches and some reversal of flow. An anomalous vascular anatomy was also observed. Computed tomography (CT) with angiography of the superior mesenteric artery showed transit of contrast from the main portal vein through a large collateral vein within the liver to the inferior vena cava. The collateral vein was approximately $1 \mathrm{~cm}$ in diameter and $3 \mathrm{~cm}$ in length (fig $2 \mathrm{~A}$ ). A coeliac and superior mesenteric artery angiogram showed that the majority of the venous flow was directed away from the liver through a large collateral vessel connecting the portal vein and the inferior vena cava with prompt filling of the right atrium (fig 2B). There was a small venous structure entering the liver during the venous phase consistent with an attenuated right portal vein. Subsequently hepatic and portal vein pressure measurements with a balloon catheter showed mean pressures of approximately $9 \mathrm{~mm} \mathrm{Hg}$ in the main portal vein, 10 $\mathrm{mm} \mathrm{Hg}$ within the shunt segment, and $4 \mathrm{~mm}$ $\mathrm{Hg}$ in the right atrium. Right hepatic vein free and wedged pressures were $7 \mathrm{~mm} \mathrm{Hg}$ and 10 $\mathrm{mm} \mathrm{Hg}$, respectively. These studies and pressure measurements clearly showed that the majority of the portal blood was bypassing the liver through the abnormal channel to the inferior vena cava. The anomalous circulation arose from the left portal vein consistent with a remnant branch of the ductus venosus in the fetal circulation.

Since this admission the symptoms of fatigue have diminished, and the pedal oedema is well controlled with diuretics. The albumin concentrations are intermittently within the normal range but his prothrombin time has remained minimally prolonged. He complains of weak feelings that are relieved by ingestion of orange juice or candy but biochemical evidence for hypoglycaemia has not been obtained. In addition, his wife reports that he occasionally develops mental status changes consistent with mild hepatic encephalopathy.

PATIENT 2

The index patient's brother, a 36 year old white man, was referred with a presumed diagnosis of alcoholic liver disease as he had a history of drinking a six pack of beer a day for 15 years. His main complaints were of fatigue, pedal oedema, and intermittent encephalopathy. On physical examination he had evidence of palmar erythema and a palpable liver but no other signs of chronic liver disease, splenomegaly, or ascites. Results of laboratory studies were as follows: white blood cell count, $6.9 \times$ $10^{9} / 1$; haemoglobin, $136 \mathrm{~g} / 1$; platelet count, 156 $\times 10^{9} / 1$; serum aspartate aminotransferase, 47 $\mathrm{U} / 1$; alanine aminotransferase, $23 \mathrm{U} / 1$; albumin, $33 \mathrm{~g} / \mathrm{l}$; total bilirubin, $47.8 \mu \mathrm{mol} / \mathrm{l}$; alkaline phosphatase, $193 \mathrm{U} / 1$; prothrombin time, 16.1 seconds; and ammonia $65 \mu \mathrm{mol} / 1$. His liver biopsy revealed micronodular cirrhosis with macrovesicular steatosis, which was attributed 
to alcohol ingestion. The discovery of his brother's vascular abnormality resulted in further work up for a patent ductus venosus. The CT scan of his liver revealed the transit of contrast from the portal vein through a large collateral vessel to the inferior vena cava and the selective superior mesenteric artery angiogram showed a vascular lesion indistinguishable from that of his brother.

PATIENT 3

The third sibling, a 40 year old man, came to the liver clinic because of the family history of a hepatic vascular anomaly observed in his brothers. He had a history of recurrent cellulitis in his lower extremities secondary to peripheral oedema of unknown origin and minimally abnormal liver function tests for over 20 years. The positive observations of the physical examination were palmar erythema, a palpable liver, and peripheral oedema. Results of laboratory studies were: a complete blood count within normal limits; serum aspartate aminotransferase, $51 \mathrm{U} / 1$; alanine aminotransferase, $24 \mathrm{U} / 1$; albumin, $35 \mathrm{~g} / 1$; total bilirubin, $41 \mu \mathrm{mol} / 1$; alkaline phosphatase, $193 \mathrm{U} / 1$; and a prolonged prothrombin time of 13.3 seconds.

The ultrasound examination revealed two mass lesions within the liver measuring $5 \times 5$ $\mathrm{cm}$ and $8 \times 12 \mathrm{~cm}$, as well as an anomalous venous communication shunting blood from the portal vein to the inferior vena cava. The CT scan confirmed the diagnosis of patent ductus venosus. An ultrasound guided liver biopsy revealed normal architecture and notably increased iron pigment within the unaffected liver. The hepatic mass lesions resembled focal nodular hyperplasia with large fibrous bands containing numerous blood vessels and bile ducts. The serum $\alpha$ fetoprotein was within normal limits, $2.7 \mu \mathrm{g} / 1$. The hepatic dry weight iron content was found to be elevated at $8745 \mu \mathrm{g} / \mathrm{g}$, suggesting the possibility of haemochromatosis. This diagnosis had previously been entertained for patient 1 prior to presentation at the clinic because of elevated concentrations of serum ferritin.

FAMILY ANALYSIS

Both parents, two sisters, a fourth brother, and the three children of patient 1 were found to have normal hepatic vascular anatomy by either ultrasound or CT scan. Both parents came from closely knit but separate communities within Louisiana and Mississippi. There were several consanguineous marriages on the father's side of the family and the brothers' paternal grandparents were first cousins.

\section{Discussion}

This report documents three brothers with a patent ductus venosus presenting in adulthood with different manifestations of liver disease. The syndrome of familial patent ductus venosus has only previously been described in three infant brothers who presented with hepatic encephalopathy and fatty degeneration of the liver. ${ }^{3}$ The angiographic appearance of the portosystemic communication in the infants $^{3}$ was anatomically indistinguishable from that observed in the adults (fig 2). Patient 1 has recently presented with evidence of mild encephalopathy, whereas patient 2, with a history of alcohol abuse, suffered from bouts of incapacitating encephalopathy. Even though the recurrent encephalopathy, histological evidence of cirrhosis, and steatohepatitis were originally attributed to alcohol ingestion, steatosis and recurrent encephalopathy are also observed in patients with patent ductus venosus. ${ }^{3-8}$

As reported in other cases with patent ductus venosus, patient 1 developed mild encephalopathy that started after the histological demonstration of progressive hepatic portal vein sclerosis. $^{10}$ In contrast, patient 3 presented without any mental status changes, and his liver biopsy revealed normal calibre portal veins in his portal triads. He also had evidence of focal nodular hyperplasia, which is associated with hypoplasia of the hepatic portal vein, as well as atrophy and hyperplasia within the hepatic parenchyma. ${ }^{9}$ In addition, both patients 1 and 3 had evidence of iron overload in either the serum or the liver. However, analysis of the HFE gene (kindly performed by Mercator Genetics, Menlo Park, California, USA) revealed that neither brother had mutations associated with haemochromatosis. ${ }^{13}$ The hepatic iron index can be elevated in patients with surgical portacaval shunting and it increases proportionately with the duration the shunt has been present. ${ }^{14}$

All three brothers suffered from pedal oedema that was attributed to the combination of marginally decreased albumin and the vascular anomaly for patients 1 and 3 and cirrhosis in patient 2 . None of the brothers had any other signs or symptoms of cardiac disease such as dyspnoea, syncope, or cyanosis and patient 1 had demonstrable right heart pressures within the normal range. Furthermore, all three brothers described having a healthy childhood. In contrast, patients with patent ductus venosus presenting in infancy or childhood often have associated cardiac defects or hypoxia due to pulmonary arteriovenous shunting. ${ }^{1-415}$

Portal venous blood contains hepatotrophic factors such as insulin, glucagon, and epidermal growth factors that are responsible for hepatocellular growth and regeneration. ${ }^{16-18}$ Hepatocytes deprived of portal venous blood atrophy and contain diminished glycogen stores leading to recurrent episodes of hypoglycaemia. Bypass of blood from the normal detoxification process within the liver can cause hyperammoniaemia and neurological disturbances. ${ }^{5}$ Portosystemic encephalopathy can manifest in adult life secondary to a congenital portacaval shunt as described by Kerlan et al in a 67 year old woman. ${ }^{7}$ In our series, only patient 2 had symptoms of severe encephalopathy with elevated ammonia concentration, whereas patient 1 suffered from mild mental status changes and experienced episodes consistent with hypoglycaemia. The latter has also been reported to occur in patients with patent ductus venosus as well as those with hypopituitarism. ${ }^{16-18}$ 
Our three patients with patent ductus venosus presented with different manifestations of disease. Patient 2 had severe encephalopathy that was thought to be consistent with the extent of his liver synthetic dysfunction and cirrhosis. Patient 3 suffered from peripheral oedema and only presented to the clinic because of his brothers' history. He was subsequently found to have focal nodular hyperplasia and mild hepatic synthetic dysfunction, whereas patient 1 presented with a complicated history including hypopituitarism, renal stones, and "idiopathic oedema". He was subsequently found to have mild synthetic dysfunction and patent ductus venosus and it is interesting to note that he developed increasing portal vein sclerosis on liver biopsy over a three year period. Thus, the degree of encephalopathy suffered may be related to both the extent of preserved liver function as well as the extent of functional shunting from the patent ductus venosus.

The management of symptoms of patients with portosystemic shunts is generally dietary and supportive. For those with deteriorating hepatic function, either surgical resection of the involved area of liver, or ligation of the shunt vessel may be required. ${ }^{137}$ Coil embolisation of the channels has also been attempted with significant improvement of encephalopathy. ${ }^{11}$ One of the main complications of surgical intervention for portosystemic shunts is acute portal venous congestion and bowel ischaemia. ${ }^{10}$ Detailed angiographic study of the portal system, including visualisation of the intrahepatic portal radicals, is suggested as a prerequisite to operation in these patients. ${ }^{10}$ Liver transplantation is also an option in the management of these patients, irrespective of their hepatic synthetic function. ${ }^{15} 19$

This report suggests a genetic basis for patent ductus venosus as an autosomal recessive trait in the brothers with a family history of consanguineous marriages. This notion is supported by report of three brothers presenting in infancy with the same venous anomaly and dogs that develop patent ductus venosus as an autosomal genetic trait. ${ }^{3420}$ Although a rare observation, patent ductus venosus should be entertained as part of the differential diagnosis for patients presenting with mild hepatic synthetic dysfunction and encephalopathy without established cirrhosis; especially those with portal vein sclerosis on biopsy. The diagnosis can be established by ultrasound or CT scan of the liver to reveal a large anomalous vessel.

1 Mitchell IM, Pollock JCS, Gibson AAM. Patent ductus venosus. Pediatr Cardiol 1991;12:181-3.

2 Morgan G, Superina R. Congenital absence of the portal vein: two cases and a proposed classification system for porta systemic vascular anomalies. 7 Pediatr Surg 1994;29. porta systen

3 Uchino T, Endo F, Ikeda S, et al. Three brothers with progressive hepatic dysfunction and severe hepatic steatosis due to a patent ductus venosus. Gastroenterology 1996;110: 1964-8.

4 Howard ER, Davenport M. Congenital extrahepatic portocaval shunts- the Abernethy malformation. 7 Pediatr Surg 1997;32;494-7.

5 Raskin NH, Price JB, Fishman RA. Portal-systemic encephalopathy due to congenital intrahepatic shunts. $N$ Engl F Med 1964;270:225-9.

6 Ohnishi K, Hatano H, Nakayama T, et al. An unusual portal-systemic shunt, most likely through a patent ductus venosus. A case report. Gastroenterology 1983;85:962-5.

7 Kerlan RK, Sollenberger RD, Palubinskas AJ, et al. Portal-systemic encephalopathy due to a congenital portocaval shunt. AfR Am F Roentgenol 1982;139:1013-15.

8 Raskin NH, Bredsen D, Ehrenfeld WK, et al. Periodic confusion caused by congenital extrahepatic portacaval shunt. Neurology 1984;34:666-9.

9 Wanless IR, Lentz JS, Roberts EA. Partial nodular transformation of liver in an adult with persistent ductus venosus. Arch Pathol Lab Med 1985;109:427-32.

10 Barsky MF, Rankin RN, Wall WJ, et al. Patent ductus venosus: problems in assessment and management. Can $\mathcal{f}$ venosus: problems in
Surg 1989;32:271-5.

11 Ohtomo K, Furui S, Saito M, et al. Enormous intrahepatic communication between the portal vein and the hepatic vein. Clin Radiol 1986;37:513-14.

12 Chagnon SF, Vallee CA, Barge J, et al. Aneurysmal portahepatic venous fistula: report of two cases. Radiology 1986;159:693-5

13 Feder JN, Gnirke A, Thomas W, et al. A novel MHC class 1-like gene is mutated in patients with hereditary haemochromatosis. Nat Genet 1996;13:399-408.

14 Adams PC, Bradley C, Frei JV. Hepatic iron and zinc concentrations after portacaval shunting for nonalcoholic concentrations after portacaval shun
cirrhosis. Hepatology 1994;19:101-5.

15 Woodle ES, Thistlethwaite JR, Emond JC, et al. Successful hepatic transplantation in congenital absence of recipient portal vein. Surgery 1990;107:475-9.

16 Starzl TE, Francavilla A, Halgrimson CG, et al. Origin, hormonal nature and action of hepatotrophic substances in portal venous blood. Surg Gynecol Obstet 1973;137:179-99.

17 Rozga J, Jepssorr B, Bengmark S. Hepatotrophic factors in liver growth and atrophy. Br F Exp Pathol 1985;66:669-78.

18 Baker AL. Hepatotrophic factors: basic concepts and clinical implications. Acta Med Scand 1985;703(suppl): 201-8.

19 Orii $\mathrm{T}$, Ohkohchi N, Kato $\mathrm{H}$, et al. Liver transplantation for severe hypoxemia caused by patent ductus venosus. $\mathcal{F}$ Pediatr Surg 1997;32:1795-7.

20 Meyer HP, Rothuizen J. Congenital portosystemic shunts (PSS) in dogs are a genetic disorder. Tijdschrift voor Diergencskunde 1991;116:80-1S. 